Indikationserweiterung für Strontiumranelat

\title{
Neue Therapieoption für morsche Männerknochen
}

- Für die Osteoporosetherapie bei Männern mit erhöhtem Frakturrisiko gibt es eine neue Therapieoption: Aktuelle Daten legen nahe, dass die Frakturprävention mit Strontiumranelat bei Männern ähnlich gut ist wie bei Frauen.

Die Osteoporosetherapie konzentriert sich in Deutschland in erster Linie auf postmenopausale Frauen. Doch sind auch viele Männer behandlungsbedürftig. Prof. Andreas Kurth, Mainz, präsentierte Prävalenzdaten, denen zufolge etwa 9,7\% der deutschen Männer über 50 Jahre eine Osteoporose haben. Das Frakturrisiko beträgt ca. $22 \%$, die Mortalitätsrate, z. B. nach einer vertebralen Fraktur, ist höher als bei Frauen. Da innovative Medikamente i.d. R. zunächst nur für Frauen zugelassen werden, können Männer nur „off-label“ behandelt werden.

\section{Erhöhte Knochenmineraldichte}

Für betroffene Patienten steht jetzt eine neue Therapieoption zur Verfügung: Seit Ende Juni ist Strontiumranelat (Protelos ${ }^{\circledR}$ ) bei der Behandlung von Männern mit Osteoporose verordnungs- und erstattungsfähig. Die Zulassungserweiterung basiert auf der multizentrischen MALEO-Studie (Kaufman JM et al. Osteoporosis Int 2012; 23 (Suppl. 2): S260), die Prof. Johann D. Ringe, Leverkusen, vorstellte. Im Vergleich zu Placebo besserte sich unter Strontiumranelat die Knochenmineraldichte (BMD) in den Lendenwirbeln $(+9,8 \%)$ und dem Femurhals $(+3,3 \%)$ binnen zwei Jahren signifikant. In der CASIMO-Studie wurde die Wirksamkeit von Strontiumranelat direkt mit der von Alendronat verglichen. Nach einem Jahr erhöhte sich die Lendenwirbel-BMD unter
Strontiumranelat um 5,8\% gegenüber Baseline (4,5\% unter Alendronat). Da der mittlere BMD-Anstieg bei Männern den für Frauen nachgewiesen Raten entspricht, könne man postulieren, dass das Medikament auch bei Männern wirkt, erklärte Ringe. Er betonte, dass Strontiumranelat im Gegensatz zu Bisphosphonaten kein rein antiresorptives Medikament sei, im Gegenteil werden Osteoblasten leicht stimuliert.

Eine Kontraindikation besteht für Patienten mit akuten oder vormaligen venösen Thromboembolien, immobilisierte Patienten und Patienten mit Überempfindlichkeitsreaktionen gegen Strontiumranelat.

\footnotetext{
- Sebastian Lux

Quelle: Pressegespräch „Osteoporose des Mannes - Indikationserweiterung PROTELOS ${ }^{\oplus ", ~ M u ̈ n-~}$ chen, September 2012 (Veranstalter: Servier)
}

\section{Akute Rhinosinusitis \\ Bewährte 5-Pflanzen-Kombination - jetzt auch mit höherer Wirkstoffkonzentration}

- Seit Anfang Oktober ist die kürzlich zugelassene 5-Pflanzen-Kombination Sinupret $^{\oplus}$ extract in Apotheken erhältlich. Sie enthält die gleichen fünf Inhaltsstoffe wie Sinupret ${ }^{\circledR}$ forte. Der Hersteller Bionorica SE nutzt für das neue Präparat ein anderes Trockenextraktverfahren, das u. a. zu einer vierfach höheren Wirkstoffkonzentration führt. "Dadurch wird eine bessere Bioverfügbarkeit und ein rascherer Wirkeintritt erreicht", sagte Bionorica SE CEO Prof. Michael Popp.

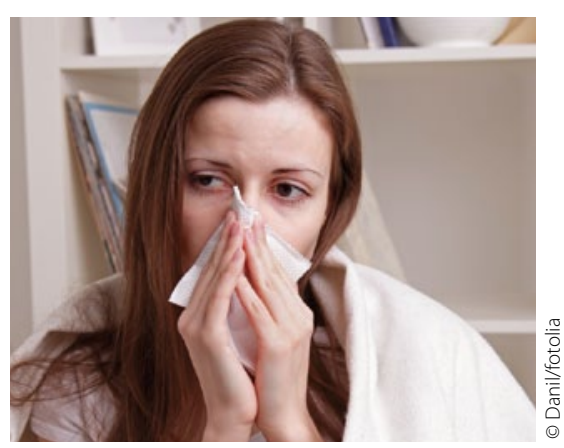

Es ist wieder Schnupfensaison. Phytotherapie kann helfen.
Das Phytopharmakon in neuer Galenik wurde in einer von Prof. Claus Bachert, Gent/Belgien, begleiteten, randomisierten, placebokontrollierten Doppelblindstudie bei 380 Patienten mit akuter Rhinosinusitis untersucht. Eingenommen wurden $480 \mathrm{mg}$ Wirkstoff, verteilt auf drei Tagesdosierungen, über 15 Tage oder die entsprechende Menge Placebodragees.

Primärer Wirksamkeitsendpunkt war die Restsymptomatik nach 15 Tagen, ermittelt mit dem Major Symptom Score, der anteriore und posteriore Rhinorrhö, nasale Verstopfung sowie Kopf- und Gesichtsschmerz berücksichtigt. In der Placebogruppe hatten die Patienten im Mittel 3,41 Punkte, in der Verumgruppe 2,38 Punkte $(p<0,0015)$.

Sonografische Zeichen für eine Rhinosinusitis hatte beim letzten Arztbesuch in der Placebogruppe noch jeder dritte Patient, in der Verumgruppe nur jeder vierte. Insgesamt werde die Abheilung der akuten Rhinosinusitis durch das Phytopharmakon im Mittel um knapp vier Tage beschleunigt.

"Eine Studie dieser Qualität ist ein Novum für Phytopharmaka", betonte Bachert. „Die Ergebnisse zeigen einen nicht nur statistisch signifikanten, sondern auch klinisch relevanten Effekt des Präparats." Das sah auch das Bundesinstitut für Arzneimittel und Medizinprodukte (BfArM) so: Das Institut hat das Präparat auf Basis dieser Studie für die Behandlung einer akuten Rhinosinusitis zugelassen.

Bachert zeigte sich zuversichtlich, dass in Kürze auch die nationalen und internationalen Leitlinien der HNO-Ärzte die 5-Pflanzen-Kombination entsprechend aufwerten. Das Phytotherapeutikum kommt als Tabletten zu je 160 mg Trockenextrakt auf den Markt. Die empfohlene Dosis liegt bei dreimal einer Tablette täglich.

- Philipp Grätzel von Grätz Quelle: Fachpresse-Konferenz zur Zulassungserweiterung von Sinupret, Berlin, September 2012 (Veranstalter: Bionorica SE) 